\title{
Nursing Intervention Program for Tuberculosis Patients by Using Epidemiological Model
}

\author{
Ebtisam Mohamed Abd El-Aal ${ }^{1}$, Manal Mansour Mostafa ${ }^{2}$ \\ ${ }^{1}$ Community Health Nursing, Faculty of Nursing, Benha University, Benha, Egypt \\ ${ }^{2}$ Community Health Nursing, Faculty of Nursing, Fayoum University, Fayoum, Egypt
}

Email address:

ebtisam_rohayem@yahoo.com (E. M. A. El-Aal)

\section{To cite this article:}

Ebtisam Mohamed Abd El-Aal, Manal Mansour Mostafa. Nursing Intervention Program for Tuberculosis Patients by Using Epidemiological Model. American Journal of Nursing Science. Vol. 4, No. 3, 2015, pp. 119-126. doi: 10.11648/j.ajns.20150403.21

\begin{abstract}
Tuberculosis is more prevalent in developing countries, in which up to $95 \%$ of cases occur. It remains a major challenge to global public health in the $21^{\text {st }}$ century. Aim: The study aimed to evaluate the effect of nursing intervention program for tuberculosis patients by using epidemiological model. Setting: The study was conducted at outpatient clinics in Benha Chest Hospital and El-Fayoum University Hospital. Sample: A simple random sample of 95 tuberculosis patients who attended to the previous mentioned settings; 45 patients from El-Fayoum University Hospital and 50 patients from Benha Chest Hospital, were selected randomly and representing 15\% of 1425 attended patients over six months in the year 2013. Tool: An interviewing questionnaire included four parts 1): Socio-demographic characteristics. 2): Patient past and present medical history. 3): Patient knowledge regarding tuberculosis. And 4): Patient knowledge about practices through asking questions related to tuberculosis, which included: a) Patient health practices regarding tuberculosis. b): Patient knowledge regarding epidemiological model as person (host), agent, and environment. Results: $84.2 \%$ of the study sample were males while $48.4 \%$ their age ranged from 20 to 40 years, and $83.2 \%$ of them were smokers. $34.7 \%$ of study sample had especial equipment compared to $85.3 \%$ of them post intervention program. Furthermore $65.3 \%$ of the study sample avoided overcrowding places pre intervention program compared to $74.7 \%$ of them post intervention program. Statistically significant differences were detected between pre and post program regarding epidemiological model. Conclusion: The present study concluded that the patients' knowledge were increased after implementing the program and their practices were improved regarding health practices and epidemiological model. Recommendations: Health education program should be provided for tuberculosis patients regarding epidemiological model during treatment phases and follow-up visits.
\end{abstract}

Keywords: Epidemiological Model, Tuberculosis Patient, Nursing Intervention

\section{Introduction}

Tuberculosis (TB) is a substantial global cause of mortality and morbidity, with 9 million new cases of active tuberculosis and 1.5 million deaths occurring in 2013. One third of the world's population is infected with Mycobacterium tuberculosis (1). And it is an important public health problem in the Eastern Mediterranean Region as reported by the World Health Organization. Every year, the disease kills 136,000 people and affects 630,000 in that region. It is considered the third most important public health problem in the Egypt (2).

Tuberculosis is a chronic disease characterized by a variable and generally long incubation period, and its etiological agent has an exceptionally wide range of hosts, including humans, domestic and wild animals, as well as high persistence in the environment (3). People suffered from the disease as well as from social branding, they were treated as 'outcasts'. Challenges with TB were not only medical, it involved the entire family; the entire economical situation of the core family changed as the key provider was no longer able to earn money. TB patients could not contribute to sustain the family, they become a burden for their family and ultimately, they were at risk to succumb to the disease (4).

An epidemiological model is usually defined as 'a logical representation of the epidemiology of disease transmission and its associated processes, and it facilitates the evaluation of the efficacy of the potential control measures and provides estimates of the future magnitude, duration and geographical extent of an outbreak given the application of specific control measures. It also provides frameworks that allow ideas about 
the behavior of a particular system to be conceptualized and communicated (5).

Nurses play a crucial role in tuberculosis control program; the International Council of Nursing (ICN) believes that nurses are in a position to advocate for strong tuberculosis control programs and to implement the elements of Directly Observed Treatment Strategy (DOTS). Nowadays, most tuberculosis treatment is carried out in the community settings; treatment is best supervised by nurses in regular contact with the patient. The nursing intervention requires a system of recommendations that improve performance and support the quality of health services (2).

\section{Aim of the Study}

To evaluate the effect of nursing intervention program for tuberculosis patient by using epidemiological model through:

1. Assessing patient knowledge and practices regarding tuberculosis and epidemiological model

2. Developing and implementing intervention program according to epidemiological model

3. Evaluating the effect of nursing intervention program of the patients' knowledge and practices regarding epidemiological model.

\section{Research Hypothesis}

Patients with tuberculosis under the study were increasing their knowledge and improving their practices regarding tuberculosis and epidemiological model, after implementing the program

\subsection{Setting}

The study was conducted at outpatient clinics in ElFayoum University Hospital and Benha Chest Hospital to collect the study data.

\subsection{Sample}

A simple random sample of 95 patients who attended to the previous mentioned settings; 45 patients from El-Fayoum University Hospital and 50 patients from Benha Chest Hospital which were selected randomly and represent $15 \%$ of 1425 patients attended over six months in the year 2013 in both settings. Under the following criterion: the patient aged more than 18 years.

\section{Tool of the Study}

An interviewing questionnaire was used to collect data which includes four parts 1): Socio-demographic characteristics as: age, sex, educational level, occupation, monthly income, and smoking. 2): Patient past and present medical history as presence of other health problems, followup, recurrent hospitalization, onset of the disease, and presence of affected person in the family. 3): Patient knowledge regarding tuberculosis as meaning of tuberculosis, signs and symptoms, incubation period, treatment, infectious period, high risk person, diagnosis.

\subsection{Scoring System}

The patients' knowledge was calculated for each item as follows: good knowledge was scored (2); while average knowledge was scored (1), and poor knowledge was scored (0). Total knowledge score was calculated to test correlations.

4): Patients' knowledge about practices as reported regarding tuberculosis which includes a): Patient health practices regarding tuberculosis as having special room, the room had good ventilation, having special equipment, taking drug regularly, making follow-up regularly, using special paper handkerchief, dispose the handkerchief safely, taking healthy diet, avoid overcrowding places.

\subsection{Scoring System}

Patients' practices were calculated for each item as follows: Regular practices were scored (2), while practices done sometimes were scored (1), and not done practices were scored (0). Total score was calculated to test correlation.

b): Patients' knowledge about epidemiological model related to person (host), agent, and environment, which included that: Person as the disease increases due to contact with other persons, disease increases with decreasing immunity, unhealthy diet increases risky for disease, healthy habits decrease infection, healthy diet decreases susceptibility to disease, physical activity decreases risk of disease, and follow-up is important. Agent as in the case of infection cause by being exposed to bacteria causing disease, microbes- activated in when lowering immunity, tuberculosis infected lung only, incubation period from 4-6 weeks, immunization effect on tuberclue bacilli, increasing disease according number of microbes, and Environment as: tuberculosis is a worldwide disease, tuberculosis spreads through air, tuberculosis can spreads through unhygienic milk, infection transmitted about sharing equipment, sanitary environment prevent spread of infection, avoid overcrowding places decreasing infection, good ventilation is important to prevents disease.

\subsection{Scoring System}

Patients' knowledge about epidemiological model was calculated for each item as follows: good knowledge was scored (2), while average knowledge was scored (1), and poor knowledge was scored (0). Total score was calculated to test correlations

Validity: Content validity was done through five experts from Faculty Members of Community Health Nursing and chest physician in chest hospital.

Reliability: Reliability coefficients were calculated for the questionnaire items. The coefficient alpha was 0.86 .

Ethical consideration: All relevant ethical aspects were considered for ensuring the confidentiality of the collected data through; gaining oral consent for participation in the study, explaining the purpose of the study, and informing 
them about their right to refuse to continue participation at any time without giving any reasons.

Pilot study: was carried out on 10 patients as $10 \%$ of the study sample to assess the tool clarity, applicability, and time needed to fill in each sheet those who participated in the pilot study were excluded from the main study sample.

\subsection{Field Work}

- $\quad$ Preparation of data collection was carried out from the beginning of May 2014 to the end of August 2014 and data collection from beginning of September 2014 to end of February 2015.

- An official letter was issued from the Dean of the Faculty of Nursing to the directors of El-Fayoum University Hospital and Benha Chest Hospital including the aim of the study to get their approval to carry out the study.

- Approval taken from the directors, an oral consent was also taken from each patient for participation.

- The researchers conducted the program twice/week (Saturdays and Thursdays), from 10.00 a.m. to 1.00 p.m. in the Outpatient clinics in each hospital

- The patients were interviewed individually by the researchers to implement the program in the outpatient clinics.

- Handout about the health education program for tuberculosis patient was provided.

\subsection{Health Educational Program Construction}

The program was conducted at four phases:

1- Preparatory phase: A review of recent, current, national and international literature in various aspects of the problem. The tools questionnaire was designed to assess the patients' knowledge and practices regarding the epidemiological model before and after implementing the program.

2- Assessment phase: The pretest questionnaire was implemented to identify the patients' knowledge about tuberculosis and their practices to prevent infections.

3- Planning and implementing phase:

The intervention program was designed, with general objective to evaluate the effect of intervention program for prevention and control of tuberculosis regarding knowledge and practices of epidemiological model

The program content included:

- Tuberculosis knowledge as: Meaning of tuberculosis, signs and symptoms, incubation period, treatment, infectious period, high risk person, and diagnosis.

- Patient practices to prevent infection and decrease the disease as having special room, the room had good ventilation, had special equipment, taking drug regularly, making follow-up regularly, using special paper handkerchief, dispose the handkerchief safely, taking healthy diet, and avoiding overcrowding places.

- Effect of epidemiological model pre and post program regarding Person as increasing disease by contacting other persons, increasing disease with decreased immunity, unhealthy diet increases risk for disease, healthy habits decreases infection, healthy diet decrease susceptibility to disease, physical activity decrease risk of disease, follow-up is important, Agent as infection caused by exposed to bacteria causing disease, microbesactivated in when lowering immunity, tuberculosis infected lung only, incubation period from 4-6 weeks, immunization effect on tuberclue bacilli, increasing disease according number of microbes, and Environment as tuberculosis worldwide disease, tuberculosis spread through air, tuberculosis can spread through unhygienic milk, infection transmitted about sharing equipment, sanitary environment prevent spread of infection, avoid overcrowding places decreasing infection, good ventilation is important to prevent disease.

The program includes five sessions 2 for theory and 3 for practice.

Each session takes from 20-30 minutes for theory and practical.

At the end of the program implemented, a booklet of the program was given to each patient as a reference.

A post test was done to evaluate the effect of the intervention program on increasing patients' knowledge and improving their practices.

The teaching methods used were discussions, brainstorming, demonstration and re-demonstration. Booklets were distributed as teaching media.

\subsection{The Fourth: Evaluating Phase}

To evaluate the effect of the health educational program of patients' knowledge and practices regarding tuberculosis by using epidemiological model through using posttest that similar to the pretest was applied.

\subsection{Statistical Design}

The collected data were analyzed and tabulated using "chi square" for number and percentage distribution, and correlation coefficient (r); was used by using the Statistical Package for Social Sciences (SPSS), version 18 to determine if there are statistically significance relations.

\section{Results}

Table (1): Showed that $84.2 \%$ of study sample were males and $48.4 \%$ of them their age ranged from 20 to 40 years. On the other hand, $69.5 \%$ of the study sample was employed while $56.8 \%$ of them were illiterate, and $83.2 \%$ of them were smokers.

Table (2): Clarified that $83.2 \%$ of the study sample were having other health problem in addition to tuberculosis, while $58.9 \%$ of the study sample were seeking follow up every two months. Although $64.2 \%$ of them were being hospitalized once, $72.6 \%$ having were suffering from the disease from less than one year.

Figure (1): Illustrated that $55.0 \%$ of the study sample gained their information from the health team and $23 \%$ of 
them gaining their information from their families and their neighbors.

Table (3): Clarified that $20 \%$ of the study sample had good knowledge regarding meaning of tuberculosis pre intervention program compared to $50.5 \%$ of them post intervention program. Results revealed also that $69.5 \%$ of study sample had good knowledge regarding infectious period pre intervention program, which improved in post intervention program to be $84.2 \%$ of them. Additionally, $61.1 \%$ of the study sample had good knowledge regarding signs and symptoms pre intervention program compared to $80.0 \%$ of them post intervention program. Over all, the results had statistically significant differences pre and post program regarding patient knowledge related to tuberculosis.

Table (4): Showed that only $34.7 \%$ of study sample had special equipment compared to $85.3 \%$ of them post

Table (1). frequency distribution of the study sample regarding their sociodemographic characteristics $(n=95)$.

\begin{tabular}{lll}
\hline Socio-demographic characteristics & No & \% \\
\hline Sex & & \\
Male & 80 & 84.2 \\
Female & 15 & 15.8 \\
Age (in years) & & \\
$<20$ year & 21 & 22.1 \\
20-40 years & 46 & 48.4 \\
$>40$ years & 28 & 29.5 \\
Education & & \\
Illiterate & 54 & 56.8 \\
Read and write & 24 & 25.3 \\
Basic education & 7 & 7.4 \\
High education & 10 & 10.0 \\
Occupation & & \\
Unemployed & 29 & 30.5 \\
Employed & 66 & 69.5 \\
Monthly income & & \\
Sufficient and saves & 2 & 2.1 \\
Sufficient & 32 & 33.7 \\
Insufficient & 61 & 64.2 \\
Smoking & & \\
Smoker & 79 & 83.2 \\
Not smoker & 16 & 16.8 \\
\hline
\end{tabular}

intervention program. As well, $62.1 \%$ of the study sample were taking all drugs regularly pre intervention program, while improved post intervention program to be $91.6 \%$. Furthermore, $65.3 \%$ of the study sample were avoiding overcrowding places pre intervention program compared $74.7 \%$ of them post intervention program. The table showed also statistically significant differences pre/post program for all practice items except having special room and the room had good ventilation $(71.6 \%$ \& $74.7 \%$ respectively).

Table (5): Clarified that there were statistically significance differences regarding epidemiological model of the disease pre/post intervention program in relation to person, agent and environment.

Table (6): Revealed that there were statistically significant differences between knowledge, practices and epidemiological model pre/post intervention program.

Table (2). Frequency distribution of the study sample regarding their past and present medical history $(n=95)$.

\begin{tabular}{lll}
\hline Past and present medical history & No & \% \\
\hline Other health problems & 79 & 83.2 \\
Follow-up & 11 & 11.6 \\
When needed & 28 & 29.5 \\
Monthly & 56 & 58.9 \\
Every two months & & \\
Recurrent hospitalization & 23 & 24.2 \\
No one & 61 & 64.2 \\
Once & 11 & 11.6 \\
Twice or more & & \\
Onset of disease (in years) & 69 & 72.6 \\
$<$ one & 18 & 18.9 \\
1-3 & 8 & 8.4 \\
$>3$ & 19 & 20.0 \\
Presence of affected person in the family & & \\
\hline
\end{tabular}

\section{Source of Information}

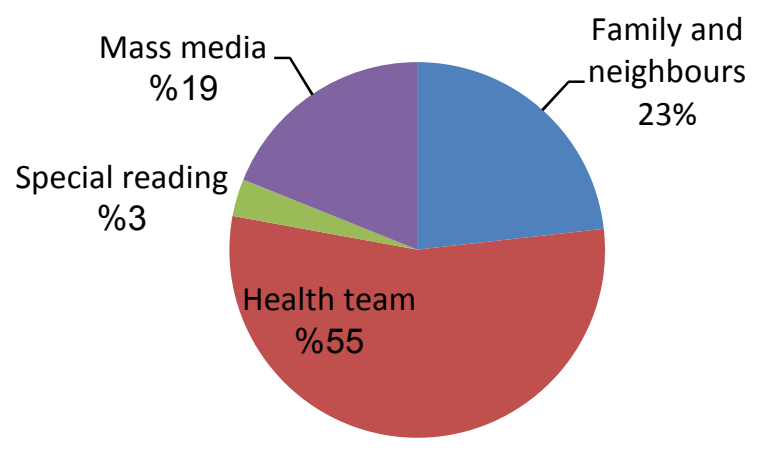

Figure (1). Frequency distribution of study sample regarding source of information. 
Table (3). Frequency distribution of study sample regarding their knowledge related to tuberculosis pre/post program (n=95).

\begin{tabular}{|c|c|c|c|c|c|c|}
\hline \multirow{2}{*}{ Patients knowledge } & \multicolumn{2}{|c|}{ Pre } & \multicolumn{2}{|c|}{ Post } & \multirow{2}{*}{$\mathbf{X} 2$} & \multirow{2}{*}{ p-value } \\
\hline & No & $\%$ & No & $\%$ & & \\
\hline \multicolumn{7}{|c|}{ Meaning of tuberculosis } \\
\hline Good & 19 & 20.0 & 48 & 50.5 & \multirow{3}{*}{19.7} & \multirow{3}{*}{0.000} \\
\hline Average & 66 & 69.5 & 39 & 41.1 & & \\
\hline Poor & 10 & 10.5 & 8 & 8.4 & & \\
\hline \multicolumn{7}{|l|}{ Signs and symptoms } \\
\hline Good & 58 & 61.1 & 76 & 80.0 & \multirow{3}{*}{9.3} & \multirow{3}{*}{0.009} \\
\hline Average & 26 & 27.4 & 16 & 16.8 & & \\
\hline Poor & 11 & 11.6 & 3 & 3.2 & & \\
\hline \multicolumn{7}{|l|}{ *Incubation period } \\
\hline Good & 27 & 28.4 & 69 & 72.6 & \multirow{2}{*}{37.1} & \multirow{2}{*}{0.000} \\
\hline Poor & 68 & 71.6 & 26 & 27.4 & & \\
\hline \multicolumn{7}{|l|}{ *Treatment } \\
\hline Good & 14 & 14.7 & 84 & 88.4 & \multirow{2}{*}{103.2} & \multirow{2}{*}{0.000} \\
\hline Poor & 81 & 85.3 & 11 & 11.6 & & \\
\hline \multicolumn{7}{|l|}{ *Infectious period } \\
\hline Good & 66 & 69.5 & 80 & 84.2 & \multirow{2}{*}{5.7} & \multirow{2}{*}{0.000} \\
\hline Poor & 29 & 30.5 & 15 & 15.8 & & \\
\hline \multicolumn{7}{|l|}{ High risk person } \\
\hline Good & 0 & 0.0 & 38 & 40.0 & \multirow{3}{*}{57.9} & \multirow{3}{*}{0.000} \\
\hline Average & 26 & 27.4 & 31 & 32.6 & & \\
\hline Poor & 69 & 72.6 & 26 & 27.4 & & \\
\hline \multicolumn{7}{|l|}{ Diagnosis } \\
\hline Good & 64 & 67.4 & 71 & 74.7 & \multirow{3}{*}{5.8} & \multirow{3}{*}{0.000} \\
\hline Average & 19 & 20.0 & 21 & 22.1 & & \\
\hline Poor & 12 & 12.6 & 3 & 3.2 & & \\
\hline
\end{tabular}

*Only two answers

Table (4). Frequency distribution of study sample regarding their reported practices regarding epidemiological model pre/post program (n=95).

\begin{tabular}{|c|c|c|c|c|c|c|}
\hline \multirow{3}{*}{ Patients practices } & \multicolumn{2}{|c|}{ Pre } & \multicolumn{2}{|c|}{ Post } & \multirow{3}{*}{$\mathbf{X}^{2}$} & \multirow{3}{*}{ p-value } \\
\hline & \multicolumn{2}{|c|}{ Respond Yes } & \multicolumn{2}{|c|}{ Respond Yes } & & \\
\hline & No & $\%$ & No & $\%$ & & \\
\hline Having special room & 81 & 85.3 & 81 & 85.3 & .04 & .84 \\
\hline Room had good ventilation & 68 & 71.6 & 71 & 74.7 & .24 & .62 \\
\hline Having special equipment & 33 & 34.7 & 81 & 85.3 & 50.5 & 0.000 \\
\hline Taking all drugs regularly & 59 & 62.1 & 87 & 91.6 & 59.9 & 0.000 \\
\hline Making follow-up regularly & 71 & 74.7 & 86 & 90.5 & 82.9 & 0.000 \\
\hline Using especial paper handkerchief & 61 & 64.2 & 72 & 75.8 & 30.8 & 0.000 \\
\hline Dispose the handkerchief safely & 73 & 76.8 & 75 & 78.9 & 59.1 & 0.000 \\
\hline Taking healthy diet & 51 & 53.7 & 93 & 97.9 & 62.8 & 0.000 \\
\hline Avoid overcrowding places & 62 & 65.3 & 71 & 74.7 & 30.6 & 0.000 \\
\hline
\end{tabular}


Table (5). Frequency distribution of study sample knowledge as reported by patients regarding epidemiological model pre/post program ( $n=95)$.

\begin{tabular}{|c|c|c|c|c|c|c|c|c|}
\hline \multirow{2}{*}{ Items } & \multicolumn{3}{|l|}{ Pre } & \multicolumn{3}{|l|}{ Post } & \multirow{2}{*}{$\mathbf{X}^{2}$} & \multirow{2}{*}{ p-value } \\
\hline & Poor & Average & Good & Poor & Average & Good & & \\
\hline \multicolumn{9}{|l|}{ Person } \\
\hline Contact increases disease with other persons & 24.2 & 11.6 & 64.2 & 3.2 & 4.2 & 92.6 & 23.5 & 0.000 \\
\hline Decreasing immunity increases disease & 77.9 & 2.1 & 20.0 & 3.2 & 10.5 & 86.3 & 110.0 & 0.000 \\
\hline Unhealthy diet increases the risk for disease & 8.4 & 27.4 & 64.2 & 3.2 & 5.3 & 91.6 & 21.0 & 0.000 \\
\hline Healthy habits decreased infection & 60.0 & 9.5 & 30.5 & 3.2 & 7.4 & 89.5 & 76.3 & 0.000 \\
\hline Healthy diet decreases susceptibility to disease & 22.1 & 22.1 & 55.8 & 1.1 & 2.1 & 96.8 & 44.3 & 0.000 \\
\hline Physical activity decreases risk of disease & 91.6 & 3.2 & 5.3 & 4.2 & 16.8 & 78.9 & 145.8 & 0.000 \\
\hline Follow-up is important & 80.0 & 7.4 & 12.6 & 1.1 & 2.1 & 96.8 & 137.3 & 0.000 \\
\hline \multicolumn{9}{|l|}{ Agent } \\
\hline $\begin{array}{l}\text { Infection caused by being exposed to bacteria causing } \\
\text { disease }\end{array}$ & 15.8 & 4.2 & 80.0 & 2.1 & 8.4 & 89.5 & 11.7 & 0.000 \\
\hline Microbes activated in when lowering immunity & 16.8 & 13.7 & 69.5 & 3.2 & 9.5 & 87.4 & 11.5 & 0.000 \\
\hline Tuberculosis infects lung only & 10.5 & 5.3 & 84.2 & 2.1 & 1.1 & 96.8 & 8.8 & \\
\hline Incubation period from 4-6 weeks & 8.4 & 14.7 & 76.8 & 3.2 & 5.3 & 91.6 & 7.7 & .02 \\
\hline Immunization has effect on tuberclue bacilli & 9.5 & 2.1 & 88.4 & 3.2 & 3.2 & 93.7 & 3.3 & .18 \\
\hline Increasing disease according number of microbes & 13.7 & 81.1 & 5.3 & 2.1 & 15.8 & 82.1 & 114.0 & 0.000 \\
\hline \multicolumn{9}{|l|}{ Environment } \\
\hline Tuberculosis is worldwide disease & 9.5 & 76.8 & 13.7 & 7.4 & 42.1 & 50.5 & 29.9 & 0.000 \\
\hline Tuberculosis spreads through air & 9.5 & 4.2 & 86.3 & 2.1 & 1.1 & 96.8 & 6.8 & .033 \\
\hline Tuberculosis can spread through unhygienic milk & 8.4 & 7.4 & 84.2 & 1.1 & 2.1 & 96.8 & 9.0 & .011 \\
\hline Infection is transmitted when sharing equipment & 3.2 & 6.3 & 90.5 & 1.1 & 1.1 & 97.9 & 4.8 & .08 \\
\hline Sanitary environment prevents spread of infection & 9.5 & 3.2 & 87.4 & 5.3 & 4.2 & 90.5 & 1.3 & .51 \\
\hline Avoiding overcrowding places decreases infection & 4.2 & 6.3 & 89.5 & 1.1 & 2.1 & 96.8 & 4.0 & .13 \\
\hline Good ventilation is important to prevent disease & 3.2 & 5.3 & 91.6 & 1.1 & 1.1 & 97.9 & 3.8 & .14 \\
\hline
\end{tabular}

Table (6). Correlations between patient knowledge and practices and epidemiological models regarding tuberculosis pre/post program.

\begin{tabular}{|c|c|c|c|c|}
\hline \multirow{3}{*}{ Items } & \multicolumn{4}{|c|}{ Knowledge } \\
\hline & \multicolumn{2}{|l|}{ Pre } & \multicolumn{2}{|l|}{ Post } \\
\hline & $\mathbf{r}$ & p-value & $\mathbf{r}$ & p-value \\
\hline Practices & 0.76 & 0.000 & 0.86 & .000 \\
\hline Epidemiological model & 0.93 & 0.000 & 0.67 & .000 \\
\hline
\end{tabular}

\section{Discussion}

Tuberculosis is a chronic infectious disease caused by Mycobacterium tuberculosis. Poverty, malnutrition and crowded living conditions have been recognized for decades to increase the risk of developing TB. In addition, lack of knowledge about TB could affect the health-seeking behavior of patients and sustain the transmission of the disease within the community (6).

Regarding socio-demographic characteristics the present study consists of nightly five patients. Their age ranged from 18 up to 40 years with a mean age of $37.17 \pm 13.53$ and the majority of the studied sample were males. This result goes in line with that of the study of Hashim et al (7), who founded that the mean age of patients in their study conducted in Iraq was $36.4 \pm 16.4$, and more than two third of them were males. The finding of Bhatt et al (8), also showed that majority of the TB patients in Thailand belong to the economically active young age group of 21-50 years. This finding is also consistent with that of Bam (9), who reported that the majority of TB patients in Nepal were in the age group of 15-54 years. As well, Getahun et al., (10), found that over $95 \%$ of new TB cases and deaths occur in low and middle-income countries.

The current study result also revealed that more than three quarters of the studied sample were smokers. This finding was consistent with study conducted by Yadav et al, (11), who mentioned that, more than two thirds of the TB patients were smoking, and their family income was insufficient. These results agree with that of Ferrara et al., (12), who clarified that smoking is a well known major risk factor associated with TB.

Considering the studied sample knowledge related to meaning and signs and symptoms, the finding of the study revealed that, there was improvement in patients' knowledge after the intervention program. This result agrees with the study conducted by Hashim et al (7), who reported in his study that the majority of the patients knew definition of TB. Also study conduct by Joshi et al. (13), showed that the majority of patients know that tuberculosis is transmitted by droplet infection, and use of materials contaminated by TB patients.

According to isolation technique, the finding of the current study revealed that, the foundation of special 
equipment for the patients was improved after implementation of the program among the majority of the study subjects. This result is congruent with WHO, (14) which stated that patient with T.B. should have special equipment. As well, this study indicated that practice of patient suffering from T.B regarding avoiding overcrowding area improved after the program, this result is supported by Wanyeki et al., (15), who identified that crowding, poor air quality within homes as a result of inadequate ventilation, and the presence of mold and smoke contribute to poor respiratory health in general and have been implicated in the spread and/or outcome of tuberculosis.

The result of the present study showed that there was improvement of epidemiological model regarding tuberculosis post intervention program related to host, agent and environment compared by pre intervention program. This result is supported by Cagri et al., (16), who mentioned that epidemiological model of tuberculosis achieve significant result in order to slow progression of T.B, and recently with Coscolla and Gagneux, (17), who stated that environmental factors such as overcrowding and poor ventilation increase exposure to infectious particles, and implementing improved ventilation has been shown to reduce $\mathrm{MTBC}$ transmission. Increasingly however, it is becoming clear that better knowledge of the bacterial determinants of virulence and their interaction with host and environmental factors will improve our understanding of the pathogenesis of TB. Similarly Kohno et al., (18), highlighted that host factors play an important role in improving the quality of clinical practice, in particular with patients who are receiving home care, and patients who have been admitted to long-term care facilities.

This study finding revealed that there were statistically significant differences between knowledge of patients regarding tuberculosis and both of practice and epidemiological model. This result is in accordance with Fikru et al., (19), who founded in their very recent study about assessment of community knowledge about Tuberculosis and its treatment in rural areas of Shashemane, Southern Ethiopia that there was a statistically significant differences regarding knowledge and practices of patients in relation to T.B. additionally Ahmed et al., (2), clarified that the main reasons for the increase of the global burden of TB are the inadequate health services, improper management practices resulting in poor case detection, misdiagnosis, and maltreatment.

\section{Conclusion}

The present study concluded that the majority of the sample under the study were male, and smoker, most of them having other health problems, and more than two thirds having the disease from less than one year. As regards, the patients' knowledge and practices the program succeeded to upgrade the patients' knowledge after implementing the program and their practices were improved regarding health practices and epidemiological model.

\section{Recommendations}

- Health education program should be provided for tuberculosis patient regarding epidemiological model during follow-up visits.

- Illustrated booklet should be provided with description of disease control and prevention.

- Further research to be carried out regarding factors affecting healthy practices for tuberculosis patients

\section{References}

[1] Ndiaye B., Thienemann F., Ota M., Landry B., Camara M., Dièye S., Dieye T., Esmail H., Goliath R., Huygen K., January V., Ndiaye I., Oni T., Raine M., Romano M., Satti I., Sutton S., Thiam A., Wilkinson K., Mboup S., Wilkinson R., and McShane H. (2015): Safety, immunogenicity, and efficacy of the candidate tuberculosis vaccine MVA85A in healthy adults infected with HIV-1: a randomised, placebo-controlled, phase 2 trial, The Lancet Respiratory Medicine, 3, (3), P.p 190-200

[2] Ahmed A., Soliman S., and Awad L. (2012): Validation of evidence-based clinical practice guideline: Nursing intervention for newly diagnosed pulmonary tuberculosis patients at community setting, Alexandria Journal of Medicine, 48, (2), P.p 155-165

[3] Ross G., De Leo G., Pongolin S., Natalini, S., Vincenzi S., and Bolzon L. (2015): Epidemiological modelling for the assessment of bovine tuberculosis surveillance in the dairy farm network in Emilia-Romagna (Italy), Epidemics 11, P.p $62-70$

[4] Wallstedt H., and Maeurer M., (2015): The History of Tuberculosis Management in Sweden, International Journal of Infectious Diseases, 32, P.p 179-182

[5] Dubé C., Garner G., Stevenson M., Sanson R., Estrada, C. and Willeberg P. (2007): The use of epidemiological models for the management of animal diseases, Conf. OIE, P.p 13-23

[6] Fikru M., Mengistu L., Tariku L., Mengistu H., and Zenebe M. (2015): Assessment of community knowledge about Tuberculosis and its treatment in rural areas of Shashemane, Southern Ethiopia. Journal of public Health. 7(3): P.p 91-97

[7] Hashim S., AL Kubaisy W., and Dulayme A. (2007): Knowledge, attitudes and Practices survey among health care workers and tuberculosis patients in Iraq., Eastern Mediterranean health journal, 24 , (4), P.p. 128- 131.

[8] Bhatt P., Bhatt B., and Shrestha B. (2009): Nepaless People's Knowledge about Tuberculosis. Saarc J. TUBER. LUNG DIS.HIV/AIDS, VI (2) 31-37

[9] Bam S. (2003): Factors affecting patients' compliance with directly observed treatment short course in Kathamndu urban areas, Nepal. Thesis submitted to Master of Primary Health Care Management, Faculty of Graduate Studies Mahidol University, Thailand. SAARC Journal of Tuberculosis, Lung disease HIV/ AIDS, VI (2): P.37.

[10] Getahun B., Ameni G., Medhin G., and Biadgilign S. (2013): Treatment outcome of tuberculosis patients under directly observed treatment in Addis Ababa, Ethiopia, The Brazilian Journal of Infectious Diseases, 7(5), P.p :521-528 
[11] Yadav P., Mathur L., and Dixit K. (2006): Knowledge and attitude towards tuberculosis among sandstone quarry workers in desert parts of Rajasthan, (Original received on 4.1.2006; Revised version received on 17.4.2006; Accepted on 16.5.2006) Indian Journal of Tuberculosis.

[12] Ferrara G., Murray M., and Winthrop K. (2012): Risk factors associated with pulmonary tuberculosis: smoking, diabetes and anti-TNF [alpha] drugs. Current Opinion in Pulmonary Medicine .18(3): P.p.233-240

[13] Joshi S., Maharjan M., and Zimmerman D. (2006): Tuberculosis awareness among TB patients visiting in DOTS clinic in Patna Hospital. SAARC, Journal of Tuberculosis, Lung Disease and HIV/AIDS; (1): 20-25

[14] World Health Organization (WHO) (2008): Tuberculosis control, surveillance, planning, financing.http://who.int/mediacentre/factsheets/fs104/en/index .html
[15] Wanyeki I., Olson S., Brassard P. (2006): Crowding, and tuberculosis in Montreal. Soc Sci Med 63; P.p 501-11.

[16] Cagri O., Amina S., Scott V. Bülent Y., Kristin P. (2012): Epidemiological models of Mycobacterium tuberculosis complex infections. Advances in Medicine 23(2): P.p77-96

[17] Coscolla, M., and Gagneux S., (2014): Consequences of genomic diversity in Mycobacterium tuberculosis, Seminars in Immunology, 26, (6); 431-444

[18] Kohno S., ImamuraY., ShindoY., Seki M., Ishida T., Teramoto S., Kadota J., TomonoK., and Watanabe A. (2013): Clinical Practice Guidelines for Nursing- and Healthcare-associated Pneumonia (NHCAP) [Complete translation] , Respiratory Investigation, 51, ( 2), P.p 103-126

[19] Fikru M., Mengistu L., Tariku L., Mengistu H., and Zenebe M. (2015): Assessment of community knowledge about Tuberculosis and its treatment in rural areas of Shashemane, Southern Ethiopia. Journal of public Health. 7(3): P.p 91-97. 